\title{
Trayectorias juveniles y factores de la demanda de educación universitaria española para el año 2009*
}

\author{
Dani Torrents** \\ Universitat Autònoma de Barcelona. Departament de Sociologia \\ danitv@hotmail.com
}

\section{Resumen}

El presente trabajo tiene como objetivo analizar la demanda de educación universitaria, sus factores y su dimensión transicional. En este sentido, se presenta una actualización del análisis de la demanda de estudios universitarios en el Estado español a partir de los datos del módulo de la EPA 2009, un módulo que nos permite analizar también a los jóvenes emancipados en relación con su origen social, lo cual nos evita perder el 33\% de la muestra. Identificamos los factores principales considerados en otras investigaciones y construimos un modelo de demanda educativa que evaluamos para el año 2009, mediante la regresión logística binaria. Se introducen, además, aspectos biográficos en el análisis para observar la importancia de la trayectoria vital en la demanda de estudios universitarios y su relación con los factores del modelo. Se observa que las desigualdades en la demanda educativa siguen presentes en 2009. Se pueden explicar por las dificultades económicas, el sexo y el origen social. La relación con el mercado de trabajo y la situación residencial representan dos aspectos que mejoran y modifican el modelo propuesto, lo que muestra la importancia de la dimensión transicional en un análisis de demanda educativa.

Palabras clave: transición; elección educativa; juventud; origen social; acceso a la universidad; enseñanza superior; jóvenes.

* Esta investigación forma parte del Plan Nacional de Investigación Científica, Desarrollo e Investigación Tecnológica (CSO2010-19271), financiado por el Ministerio de Ciencia e Innovación, con el título de Itinerarios universitarios, equidad y movilidad ocupacional. Asimismo, sintetiza los hallazgos más importantes de la tesina de máster El acceso a la universidad en el estado español en 2009: Los condicionantes en el acceso y la dimensión transicional.

** Investigador predoctoral becado concedido por el Ministerio de Educación, Cultura y Deporte, en el marco del Programa de Formación de Profesorado Universitario 2012 (FPU). 
Abstract. Youth pathways and factors of Spanish univeristy demand for 2009

This paper aims to analyze the demand for university studies, its factors and its transitional dimensions. We present an updated analysis of university studies demand in Spain with data from the EPA module of 2009. This module allows analyzing independent youth jointly with their social background, thus avoiding losing $33 \%$ of the sample. We identify the main factors considered in previous research, and build a model of educational demand evaluated for 2009 during the economic crisis using binary logistic regression. Biographical aspects are also introduced in the analysis to observe the importance of youth pathways in the demand for university studies and their relation with the model factors. It is noted that inequalities in educational demand are still present in 2009, which are explained by economic difficulties, gender, and social background. The relationship with the labour market and housing situation are two elements that improve and modify the proposed model of educational demand, showing the relevance of a transitional dimension.

Keywords: transitional dimension; educational choice; youth; social background; university access; youth; higher education.

\section{Sumario}

1. Introducción: la universidad como factor de movilidad social

2. Revisión teórica 3. Metodología
4. Resultados

5. Conclusiones

Referencias bibliográficas

\section{Introducción: la universidad como factor de movilidad social}

Diferentes estudios han puesto el acento en el papel que desempeña la universidad como factor de movilidad social en términos de inserción laboral y enclasamiento (Carabaña, 2005; Planas y Fachelli, 2010). Nadie pone en duda que los individuos que pasan por la universidad gozan de mejores posiciones socioeconómicas respecto a los que prefieren otras vías formativas, al menos en términos de probabilidades, y que el hecho de obtener un título universitario ha estado menos relacionado con la realización de trabajos manuales no cualificados en el futuro (Soler et al., 2008), más asociados a tasas de paro, precarización, salarios bajos y peores condiciones de trabajo. Además, en Planas y Fachelli (2010) y en Fachelli et al. (2014), se muestra que la inserción laboral y social de las personas graduadas está poco condicionada por filtros sociales, es decir, la universidad discrimina poco por razones de origen familiar en su tarea de elevación del nivel de estudios de la población y en la movilidad social, una vez se ha accedido al sistema de educación superior.

No obstante, aunque podríamos afirmar que la universidad representa un motivo de ascenso social para gran parte de las personas que acceden a ella, el hecho es que, en efecto, no todas presentan la misma probabilidad 
Tabla 1. Probabilidades de demanda de estudios universitarios y composición social de la universidad según el nivel educativo de origen* (cohorte 1983-1986 en 2009)

\begin{tabular}{lcccc}
\hline & $\begin{array}{c}\text { Nivel educativo } \\
\text { de los padres bajo }\end{array}$ & $\begin{array}{c}\text { Nivel educativo de } \\
\text { los padres medio }\end{array}$ & $\begin{array}{c}\text { Nivel educativo de } \\
\text { los padres alto }\end{array}$ & Total \\
\hline Perfil de los universitarios $^{* *}$ & $12,5 \%$ & $50,7 \%$ & $36,8 \%$ & $100 \%$ \\
Probabilidad de acceso*** $^{\text {Pros }}$ & $12,3 \%$ & $30,8 \%$ & $66,6 \%$ & $31,1 \%$ \\
\hline
\end{tabular}

* Los diferentes niveles educativos se clasifican del siguiente modo: estudios primarios o inferiores (nivel educativo bajo), secundaria obligatoria, postobligatoria o formación profesional (nivel educativo medio) y estudios universitarios (nivel educativo alto).

** Incluye graduados y estudiantes universitarios en 2009.

*** La probabilidad de demanda de estudios universitarios se calcula como el porcentaje de individuos que han accedido a la universidad respecto al total de cada subgrupo social.

Fuente: elaboración propia a partir de datos del módulo de la EPA 2009.

de acceder $^{1}$. Parece ser que la desigualdad se produce, sobre todo, en el momento de entrar, que es cuando intervienen importantes filtros sociales. Concretamente, alrededor de un 30\% de los jóvenes de una misma generación demandan estudios universitarios, tanto a nivel español como a nivel catalán, sólo en sus edades más jóvenes (Merino y García., 2007), y esta proporción ha tendido a aumentar año tras año, pero, ¿quiénes constituyen este $30 \%$ ? A nivel estatal, de la generación nacida entre el 1983 y el 1986 (de 22 a 25 años en 2009), sólo el 12\% del total de individuos de origen familiar con nivel educativo bajo lo ha conseguido, frente al $67 \%$ de individuos de origen familiar con nivel educativo alto (tabla 1). Como se puede apreciar, la probabilidad de acceder a estudios universitarios presenta diferencias según el origen social, lo que indica la existencia de importantes barreras que actúan en el momento en que el individuo elige si demandar o no estudios universitarios.

El análisis de la demanda de estudios universitarios remite a un tema no menos complejo: el de la equidad educativa y la equidad social. No todos los jóvenes presentan la misma probabilidad de pasar por el sistema universitario y, por lo tanto, de acceder a una ocupación de alta cualificación. Analizar por qué los jóvenes demandan un nivel educativo es una cuestión clave para poder entender la evolución de la equidad social, especialmente en un contexto de crisis económica y de cambios sociales constantes.

Varias investigaciones han planteado un análisis de los factores que actúan en la demanda de estudios universitarios y, aunque existe un consenso relativo en los modelos utilizados, encontramos discrepancias o ausencias de alguno de ellos en función de los autores, del período analizado y de la técnica de análisis empleada (De Pablos y Gil, 2007). A continuación, nos proponemos realizar una revisión de la literatura existente, a fin de identificar los factores clave y construir un modelo de elección educativa capaz de dar cuenta de la demanda de estudios universitarios para el año 2009. A partir del análisis de

1. Interesante la analogía de Carabaña (2005), que se refiere a la universidad como un autobús con un mismo destino, pero en el cual no todos pueden subir. 
estos factores, podemos entender el porqué de los diferenciales que justamente hemos apuntado, los cuales muestran que la demanda universitaria no es aún equitativa entre los diferentes grupos sociales.

\section{Revisión teórica}

\subsection{Los factores de la demanda de educación universitaria}

Entendemos la demanda de educación como un proceso de elección individual de acudir a un nivel educativo determinado. En este proceso de elección, actúan una serie de factores que la condicionan. Como hemos observado, la elección de demandar un nivel educativo no es, en ningún caso, aleatoria o espontánea entre la población, sino que se utilizan ciertas informaciones o elementos para ello, sea esta elección más o menos consciente y deliberada. El análisis de la demanda educativa ha ofrecido diferentes respuestas, que van desde posturas funcionalistas o de reproducción social (Bourdieu y Passeron, 1990; Bernstein, 1988; Baudelot y Establet, 1987), hasta orientaciones de tipo más intencionalista, como es la teoría del capital humano (Becker, 1975; Kodde, 1986). Sin entrar en detalle en ellas, estas teorías constituyen la base que dota de contenido a los diferentes factores que se han analizado en la literatura.

Algunos estudios han puesto de relieve distintos elementos que discriminan en la demanda de estudios universitarios (Mora, 1997; Albert, 2000; González y Dávila, 1998; Carabaña, 2004; Rahona, 2006; De Pablos y Gil, 2007; Gil et al., 2010) y podemos hablar de cierto consenso en algunos de los tratados. A partir de ellos, y de acuerdo con las variables de que disponemos, construiremos el modelo de análisis que vamos a utilizar. A continuación, los describiremos explicando qué papel desempeñan como condicionantes y veremos qué resultados han obtenido en investigaciones precedentes:

a) Menores en el hogar: se parte de la premisa de que los menores en el hogar comportan una carga económica (Behrman y Taubman, 1989) y que, en este sentido, la existencia de ellos puede representar un indicador de los recursos económicos disponibles. De acuerdo con la teoría del capital humano, cuantos menos recursos tenga el individuo o la familia, más reducida resulta su capacidad para afrontar una inversión educativa. Se presume, pues, una relación entre la existencia de menores en el hogar y una demanda inferior de estudios universitarios, como han podido observar Peraita y Sánchez (1998), Rahona (2006) y De Pablos y Gil (2007).

b) Nivel formativo de los padres y de otros miembros del hogar: Rahona (2006 y 2009) describe tres relaciones causales entre el nivel formativo de los padres o de otros miembros del hogar y la demanda de estudios universitarios. La primera representa una explicación bourdieuana relacionada con el capital cultural. Se afirma que el nivel educativo de los padres constituye el capital cultural que se transmite a los hijos, entendiendo por ello sus habilidades cognitivas y culturales. Otra dimensión cultural considera el nivel formativo 
del hogar como una influencia en las preferencias de los jóvenes y, como sostienen Bowles y Gintis (2002), una transmisión intergeneracional de la situación económica y formativa de los padres.

c) Los condicionantes derivados del nivel formativo de los padres u otros miembros del hogar se pueden cristalizar en aspectos objetivos (cualificaciones insuficientes para acceder a un nivel educativo superior, al presentar menor habilidad) o en aspectos subjetivos (creencias o percepciones de sus habilidades), todo ello ligado a la perspectiva de Boudon (Jackson, 2013). La mayoría de los estudios analizados destacan la importancia del nivel formativo de los progenitores en la demanda de educación universitaria. Calero (1996) también observa esta relación por lo que a otros miembros del hogar se refiere (hermanos, tíos, abuelos, etc.), aunque de forma menos acentuada que en el caso de los progenitores.

d) Nivel de renta del hogar: este factor constituye el indicador por excelencia de los recursos disponibles en el hogar. Se ha evidenciado una relación positiva entre el nivel de renta y la demanda de estudios universitarios (González y Dávila, 1998). Hay que decir, no obstante, que no en todas las bases de datos encontramos una variable referente al nivel de renta, por este motivo, se utiliza la máxima categoría ocupacional o socioeconómica del hogar como variable proxy respecto a las principales características económicas del hogar (Fachelli, 2009; Rahona, 2009). Este es nuestro caso.

e) Proporción de desempleados en el hogar: representa otro indicador de los recursos económicos disponibles en una familia. La proporción se entiende como el número relativo de personas en edad de trabajar que están desempleadas respecto al total de personas en edad de trabajar. Aunque no sea un indicador definitivo de los recursos económicos disponibles en el hogar, nos ofrece una visión de la relación que existe con el mercado de trabajo. Albert (2000) define dos posibles efectos de esta variable en la demanda de estudios universitarios: por un lado, puede haber un efecto renta, a mayor proporción de desempleados, menores recursos y, por tanto, menor demanda de nivel educativo superior. Por otro lado, puede haber un efecto de sustitución, en el sentido de que, a mayor proporción de desempleados, menor preferencia por el mercado laboral y, por tanto, mayor demanda de estudios universitarios. Tanto Albert (2000) como De Pablos y Gil (2007) obtienen un efecto renta.

f) Sexo: las mujeres tienden a acceder a estudios universitarios en mayor proporción que los hombres (González y Dávila, 1998; Ariño y Llopis, 2011). Esto podría explicarse por el hecho de que ellas tienen unas tasas de paro más elevadas y menos coste de oportunidad de seguir estudiando (Albert, 2000). Además, Mora (1997) y Merle (2009) afirman que las mujeres necesitan un nivel educativo superior para poder competir con los hombres en el mercado laboral. Otras perspectivas atribuyen este hecho a diferentes expectativas y motivaciones derivadas de procesos de socialización diferentes (López, 1995). La mayoría de los estudios realizados sobre el acceso a 
la universidad contemplan esta variable y la confirman como significativa (De Pablos y Gil, 2007).

g) Tamaño del hogar: se presupone que, cuanto más grande sea un hogar, en referencia al número de miembros que conviven en él, menores son los recursos de los que disponen y, por lo tanto, menos demanda educativa se produce. Esta idea la sostienen, entre otros, Carabaña (2004) y Escribà (2006), pero centrándose principalmente en la cantidad de hermanos. Si bien el tamaño del hogar no tiene por qué aumentar únicamente con los hijos e hijas (hermanos) inactivos, sino que pueden ser otros familiares o hijos o hijas ocupados, es interesante poder discernir si este factor explica o no variaciones en la demanda de educación universitaria. Nosotros no sólo nos centraremos en el número de hermanos, sino también en la cantidad de personas que conviven en el ámbito doméstico, como familiares y cónyuges.

Estos serán los diferentes factores que se incluirán en nuestro modelo de demanda educativa, donde trataremos de comprobar si los resultados previos se constatan para el año 2009. El peso de la etnia del individuo en la demanda de educación universitaria no ha sido central en los análisis en el Estado español, ya sea porque el nivel de inmigración no empezó a incrementarse hasta comienzos del siglo xxI, y los efectos más importantes en el acceso a la universidad los encontraríamos años más tarde (especialmente cuando la inmigración de segunda generación tenga la edad necesaria para acceder a la universidad), ya sea por la naturaleza de los datos disponibles. Este dato tampoco se utilizará aquí, aunque sería interesante para estudios futuros.

\subsection{La universidad dentro de las trayectorias juveniles}

La demanda de estudios universitarios representa una elección dentro de algunos de los itinerarios juveniles y se adapta a la elección que mejor responde a los deseos y a los recursos del individuo en cada momento y situación (Furlong et al., 2006). La perspectiva de la transición que sustenta este modo de entender la juventud y sus elecciones nos subraya la importancia del contexto y de la situación de la persona en cada momento de escoger a la hora de analizar correctamente su comportamiento (Casal et al., 2006a; Pollock, 2009). No todos los jóvenes potenciales a matricularse en estudios universitarios se encuentran en la misma situación biográfica, sino que lo hacen dentro de trayectorias juveniles diferentes. Por lo tanto, es necesario tener en cuenta estos aspectos para no desligar el análisis de los factores que llevan a una elección, de la situación en la que se encuentran las personas que realizan dicha elección. Desafortunadamente, no siempre se ha tenido en cuenta en análisis parecidos al que aquí se realiza.

La situación biográfica del individuo influye en sus expectativas de futuro e incluso en sus opciones posibles de acción en cada momento. Es importante tener en cuenta que existe una articulación de doble dirección entre las eleccio- 
nes que se realizan y la situación dentro de una trayectoria juvenil, ya sea que la situación biográfica condicione las elecciones tomadas o que las elecciones tomadas limiten la situación biográfica del individuo. Dicho de forma más clara: la persona que está trabajando a jornada completa puede que tenga un interés menor o menos oportunidades de demandar estudios universitarios, lo cual condiciona su elección, o bien la persona que ha decidido demandar estudios universitarios puede presentar menores incentivos o capacidades para insertarse laboralmente a jornada completa, lo cual limita su situación biográfica y actúa independientemente de los otros factores comentados. Sea cual sea la dirección, se pone de relieve la articulación entre ambos elementos, lo que obliga a considerar aspectos transicionales en cualquier análisis de demanda educativa.

Partiendo de esta consideración, en el presente trabajo, nos proponemos analizar si la situación biográfica tiene un peso importante en la demanda de estudios universitarios, con lo cual complementamos el modelo de factores que se han tratado tradicionalmente y que hemos enumerado en el epígrafe anterior. Concretamente, nos centramos en dos aspectos distintos: la situación del individuo en la transición entre la educación y el mercado laboral, y la situación residencial, es decir, el paso de la convivencia con los progenitores en un mismo hogar, a la emancipación residencial.

Por un lado, nos preguntamos si la presencia en el mercado de trabajo está relacionada con la demanda de educación, pues es de suponer que una persona con una relación más fuerte con el mundo laboral tenga unas expectativas de futuro diferentes a las que no presentan relación alguna con él: el modo como el individuo ha ido construyendo su vida laboral y educativa limita su abanico de elecciones futuras, sus intenciones y sus capacidades (Staff, 2007). Este aspecto es de extrema importancia para que otros condicionantes no sean sobredimensionados o malinterpretados si se analizan aisladamente, por ejemplo: cabe esperar que las expectativas laborales o los recursos disponibles y su efecto en la demanda educativa varíen según la relación que presenten con el mercado de trabajo.

Por otro lado, la situación en la transición residencial también puede tener un impacto en la intensidad y en la variabilidad de los efectos de los demás factores considerados. Los recursos disponibles, las expectativas de futuro o las motivaciones pueden presentar pesos distintos según la situación residencial del individuo: la persona que vive sola puede mostrar un mayor peso de los condicionantes económicos que la que vive aún con sus padres, al no tener el soporte directo de la familia. Del mismo modo, también el hecho de emanciparse podría presentar una relación con las diferentes motivaciones educativas de los individuos.

En resumen, creemos que introducir esta perspectiva de transiciones en el análisis de la demanda de educación universitaria enriquecerá los hallazgos, al considerar la población no como una realidad homogénea, sino como un conjunto de situaciones biográficas distintas, con efectos diferentes sobre la demanda educativa. 


\section{Metodología}

\subsection{Modelo de análisis}

Este artículo se divide en dos partes: por un lado, se propone hacer una revisión de los factores que se consideran relevantes en la demanda de educación universitaria para el año 2009 a nivel español y, por otro lado, la incorporación de la perspectiva biográfica en el análisis de la demanda educativa, mediante la introducción de factores referentes a la trayectoria juvenil.

La primera parte la compone lo que llamaremos modelo teórico inicial, de acuerdo con los diferentes elementos que hemos enumerado anteriormente. Nos proponemos analizar si los factores discriminantes en otros estudios se observan como significativos para el año 2009. Este modelo teórico inicial está formado por diferentes hipótesis, cada una de las cuales persigue la finalidad de determinar si el factor analizado es o no discriminante en la explicación dela demanda de educación universitaria. Las diferentes hipótesis se recogen en la tabla 2.

En cuanto a la segunda parte del análisis, introducimos dos factores que hacen referencia a la situación biográfica del individuo (tabla 3). Ello nos permite tener en cuenta en el análisis los aspectos relacionados con la situación y el contexto vivencial en el que se encuentra. Podemos observar cómo estos elementos que nos indican en qué contexto se encuentra el individuo influyen, modifican o eliminan los efectos de los demás factores.

El primero de los elementos aborda la cuestión laboral de los jóvenes mediante un indicador de la transición de la escuela al trabajo. La hipótesis afirma que una relación fuerte con el mercado de trabajo constituye un condicionante negativo de la demanda de estudios universitarios. Si se observa una influencia nula entre la relación con el mercado de trabajo y la demanda de estudios universitarios, el resultado estará mostrando una gran flexibilidad y adaptabilidad del sistema educativo en relación con el mundo laboral, así como una buena configuración del sistema universitario para adaptarse a las necesidades de algunos jóvenes con trayectorias laborales diferentes, y no condicionar la compaginación entre el trabajo y los estudios. En cambio, si se confirma una diferencia en la demanda de educación universitaria según la situación laboral, ello puede estar indicando una fuerte rigidez del sistema universitario para adaptarse al mercado de trabajo, lo que dejaría fuera a algunos perfiles de jóvenes, además de establecer una diferencia entre las motivaciones de los individuos respecto a sus estudios, en función de su situación laboral.

El otro factor que planteamos en el análisis se refiere a la situación residencial de la persona. Esta hipótesis sostiene que, cuando dicha circunstancia se presenta bajo diferentes formas, se producen variaciones en los efectos de los condicionantes considerados en la elección educativa, puesto que los recursos, las motivaciones o las expectativas pueden verse modificados según la situación residencial. A diferencia del primer factor comentado, que lo consideraremos como uno más dentro del modelo de demanda, esta dimensión residencial la utilizaremos como variable de control, observando si es discriminante en 
Tabla 2. Hipótesis del modelo teórico inicial

\begin{tabular}{lll}
\hline & \multicolumn{1}{c}{ Factores } & \multicolumn{1}{c}{ Hipótesis según el marco teórico } \\
\hline H1.1 & Menores en el hogar & $\begin{array}{l}\text { A mayor número de menores en el hogar, menor } \\
\text { demanda educativa universitaria. }\end{array}$ \\
\hline H1.2 & Nivel formativo de los padres & $\begin{array}{l}\text { Las personas que demanden estudios universitarios } \\
\text { tendrán un origen formativo mayor. }\end{array}$ \\
H1.3 & Nivel formativo de otros miembros & $\begin{array}{l}\text { Las personas que convivan con otras personas con } \\
\text { el hogar } \\
\text { deludios universitarios demandarán más educación } \\
\text { universitaria. }\end{array}$ \\
\hline H1.5 & $\begin{array}{l}\text { Nivel de renta del hogar } \\
\text { (categoría socioeconómica) }\end{array}$ & $\begin{array}{l}\text { Si la categoría socioeconómica del hogar es alta, } \\
\text { habrá mayor demanda de estudios universitarios. }\end{array}$ \\
\hline en el hogar & $\begin{array}{l}\text { Cuanto mayor sea la proporción de desempleados } \\
\text { en el hogar, menor demanda de estudios } \\
\text { universitarios se producirá (efecto renta). }\end{array}$ \\
\hline H1.6 & Sexo & $\begin{array}{l}\text { Las mujeres demandarán educación universitaria } \\
\text { en mayor proporción que los hombres. }\end{array}$ \\
& Tamaño del hogar & $\begin{array}{l}\text { Cuanto mayor sea el tamaño del hogar, menor } \\
\text { demanda de educación universitaria habrá. }\end{array}$ \\
\hline
\end{tabular}

Fuente: elaboración propia.

Tabla 3. Hipótesis de los factores transicionales

\begin{tabular}{lll}
\hline & \multicolumn{1}{c}{ Factores } & \multicolumn{1}{c}{ Hipótesis } \\
\hline H2 & Situación laboral & $\begin{array}{l}\text { Las personas que presentan mayor relación } \\
\text { con el mercado de trabajo demandarán educación } \\
\text { universitaria en menor medida. }\end{array}$ \\
\hline H3 & Situación residencial & $\begin{array}{l}\text { Los factores relevantes en la elección educativa } \\
\text { cambiarán según la situación residencial. }\end{array}$ \\
\hline
\end{tabular}

Fuente: elaboración propia.

cuanto al efecto de los demás factores. Hay que aclarar que la emancipación que aquí consideramos es puramente residencial y no implica necesariamente una emancipación familiar real.

\subsection{Diseño de análisis}

Los datos con los que trabajamos son los recogidos en el módulo de la Encuesta de Población Activa (EPA) de 2009 sobre la incorporación de la juventud al mercado laboral (Instituto Nacional de Estadística, 2010). La investigación consta de una muestra de 2.713 individuos nacidos entre el 1983 y el 1986 (de 22 y 25 años en 2009), residentes en el Estado español y que potencialmente pueden acceder a la universidad. Fijamos el límite inferior en 22 años, porque ello nos permite garantizar que la mayoría de los individuos tienen la edad suficiente para haber entrado en la universidad, con ello recogemos aquellos 
casos de trayectorias educativas prolongadas o complejas, cada vez más comunes entre la juventud (Casal, 2006b) ${ }^{2}$.

En este módulo, obtenemos una ligera ventaja informativa, a diferencia de las EPAs ordinarias y de otras bases de datos utilizadas para el análisis de los factores de la demanda de estudios universitarios en el Estado español. Se pregunta a los jóvenes por el nivel académico de sus padres, vivan o no con ellos, hecho que nos permite incluir en el análisis a esas personas que no viven con sus padres, pero de las cuales nos interesa conocer su origen familiar. La respuesta, pues, no depende de la convivencia en un mismo hogar. Esto es importante, ya que un 33,5\% de los individuos encuestados entre 22 y 25 años, con fuertes diferencias entre clases sociales - los de origen social bajo, más-, no viven con sus padres, lo que provoca que los perdamos en una EPA ordinaria al introducir el origen social en el modelo de demanda.

Nuestra variable dependiente es la demanda de educación superior entendida como variable dicotómica (codificada con valores 0 y 1 ), donde 0 implica que no demandó y 1, que sí lo hizo. El diseño de la investigación que aquí se describe no entrará, pues, en aspectos cualitativos de esta demanda, sobre si ha significado egreso o sobre si se da en una u otra titulación. Entendemos la demanda como deseo cumplido y dejamos fuera posibles demandas de educación universitaria que no han llegado a cristalizarse, lo que sería motivo de otro análisis también interesante. La elección académica se analizará a partir de un modelo de regresión logística binaria, que nos permite analizar una variable dependiente $Y i$ que incluye dos alternativas excluyentes en términos de probabilidades (demanda y no demanda), a partir de las variables independientes (López-Roldán y Lozares, 2000).

Es importante remarcar qué entendemos por demanda de estudios universitarios. Se construye el indicador de la demanda universitaria mediante el cruce de dos variables: $a$ ) el nivel de estudios obtenido y $b$ ) el nivel de estudios cursados en el momento de realizar la investigación. Por lo tanto, se considera como demanda de estudios universitarios aquellos individuos de la cohorte que sabemos que han pasado por la universidad. Por otro lado, la «no demanda» se entiende como aquellos individuos que no tienen ni realizan estudios universitarios, pero que sí han alcanzado un nivel de educación secundaria superior (necesario para acceder a la universidad) ${ }^{3}$. De este modo, se compara la solicitud de educación universitaria con los individuos que pueden realizarla potencialmente, pero que no lo han hecho.

Finalmente, en la tabla 4, presentamos la relación de variables disponibles en la EPA 2009, que serán consideradas en el análisis como indicadores del modelo de demanda planteado.

2. Como muestra el INE, la media de acceso a la universidad en 2002 (Velázquez, 2004) es de 22,3 años, con tendencia a aumentar, un dato que se aleja bastante de la edad teórica de entrada al sistema universitario (17-18 años).

3. Incluyen bachillerato, grado superior de formación profesional, programas de formación que precisan estudios secundarios de segunda etapa y programas de formación que precisan estudios de grado superior. 
Tabla 4. Tabla de variables disponibles en la EPA 2009 consideradas en el análisis

\begin{tabular}{|c|c|c|c|c|}
\hline \multirow[b]{2}{*}{ Indicadores } & \multirow[b]{2}{*}{ Categorías } & \multicolumn{3}{|c|}{$\begin{array}{l}\text { Proporción/media* } \\
\text { de la población }\end{array}$} \\
\hline & & Total & $=1$ & $=0$ \\
\hline \multicolumn{5}{|l|}{ Variable dependiente } \\
\hline Elección de estudios universitarios & $\begin{array}{l}\text { 1) Demanda } \\
\text { 0) No demanda }\end{array}$ & $\begin{array}{l}42,3 \% \\
57,7 \%\end{array}$ & $\begin{array}{r}100 \% \\
-\end{array}$ & $100 \%$ \\
\hline \multicolumn{5}{|l|}{ Modelo teórico inicial } \\
\hline $\begin{array}{l}\text { Proporción de desempleados }>\text { de } 16 \\
\text { años en el hogar, respecto al total de } \\
\text { miembros }>\text { de } 16 \text { años. }\end{array}$ & Numérica & 0,11 & 0,08 & 0,14 \\
\hline Menores de 16 años en el hogar & $\begin{array}{l}\text { 1) Sí } \\
\text { 0) No }\end{array}$ & $\begin{array}{l}15,1 \% \\
84,9 \%\end{array}$ & $\begin{array}{r}8,9 \% \\
91,1 \%\end{array}$ & $\begin{array}{l}23,4 \% \\
76,6 \%\end{array}$ \\
\hline Tamaño del hogar (miembros) & Numérica & 2,63 & 2,75 & 2,47 \\
\hline $\begin{array}{l}\text { Categoría socioeconómica del hogar } \\
\text { (máxima de los miembros) }^{1}\end{array}$ & $\begin{array}{l}\text { 1) Profesionales y dir. } \\
\text { 2) Administrativos } \\
\text { 3) Cuenta propia } \\
\text { 4) Obreros y agricultores }\end{array}$ & $\begin{array}{r}46,9 \% \\
23,0 \% \\
6,7 \% \\
23,4 \%\end{array}$ & $\begin{array}{r}62,4 \% \\
18,9 \% \\
6,4 \% \\
12,3 \%\end{array}$ & $\begin{array}{r}26,4 \% \\
28,4 \% \\
7,2 \% \\
38,1 \%\end{array}$ \\
\hline Máximo nivel educativo de los padres & $\begin{array}{l}\text { 1) Sin estudios o prim. } \\
\text { 2) Secundarios } \\
\text { 3) Superiores }\end{array}$ & $\begin{array}{l}17,5 \% \\
54,7 \% \\
27,8 \%\end{array}$ & $\begin{array}{l}12,5 \% \\
50,7 \% \\
38,8 \%\end{array}$ & $\begin{array}{l}24,7 \% \\
60,5 \% \\
14,8 \%\end{array}$ \\
\hline $\begin{array}{l}\text { Otros miembros del hogar con } \\
\text { estudios superiores }\end{array}$ & $\begin{array}{l}\text { 1) Sí } \\
\text { 0) No }\end{array}$ & $\begin{array}{l}31,7 \% \\
68,3 \%\end{array}$ & $\begin{array}{l}41,3 \% \\
58,7 \%\end{array}$ & $\begin{array}{l}18,6 \% \\
81,4 \%\end{array}$ \\
\hline Sexo & $\begin{array}{l}\text { 1) Hombre } \\
\text { 0) Mujer }\end{array}$ & $\begin{array}{l}44 \% \\
56 \%\end{array}$ & $\begin{array}{l}40,2 \% \\
59,8 \%\end{array}$ & $\begin{array}{l}49,1 \% \\
50,9 \%\end{array}$ \\
\hline \multicolumn{5}{|l|}{ Variables transicionales } \\
\hline Situación de residencia & $\begin{array}{l}\text { 1) Vive con los padres } \\
\text { 0) No vive con los padres }\end{array}$ & $\begin{array}{l}73,4 \% \\
26,6 \%\end{array}$ & $\begin{array}{l}85,4 \% \\
14,6 \%\end{array}$ & $\begin{array}{l}56,8 \% \\
43,2 \%\end{array}$ \\
\hline Situación con el mercado laboral & $\begin{array}{l}\text { 1) Trabaja a } t / c \\
\text { 2) Trabaja a t/p } \\
\text { 0) No trabaja }\end{array}$ & $\begin{array}{l}41,3 \% \\
14,4 \% \\
44,4 \%\end{array}$ & $\begin{array}{r}30 \% \\
17,4 \% \\
52,6 \%\end{array}$ & $\begin{array}{l}56,6 \% \\
10,3 \% \\
33,1 \%\end{array}$ \\
\hline
\end{tabular}

* Valores para nuestra población de análisis (universitarios y universitarios potenciales nacidos entre 1983 y 1986).

1. Se ha utilizado una clasificación de las categorías socioeconómicas que ofrece el INE (CSE), en cuatro niveles (entre paréntesis, los valores de la EPA): profesionales y directivos $(4,6,10,11$ y 12$)$, administrativos (13 y 18), cuenta propia (7, 8 y 9$)$ y obreros y agricultores $(1,2,3,5,14,15,16$ y 17$)$.

Fuente: elaboración propia a partir de datos de la EPA.

\section{Resultados}

\subsection{Factores de demanda de educación universitaria para 2009}

Todas las variables consideradas en el modelo teórico inicial (modelo 1) influyen en el acceso a la universidad.

Un factor con un fuerte impacto en la demanda es la categoría socioeconómica del hogar. Se reducen en un $75 \%$ las probabilidades de demanda universitaria en el caso de individuos de categoría socioeconómica obrera o agricultora, respecto a directivos o profesionales. 
La proporción de desempleados y la presencia de menores de 16 años en el hogar son factores que influyen negativamente en el acceso a la universidad: aquellos individuos que viven en domicilios con mayor proporción de desempleados y con menores de 16 años presentan menos probabilidades de acceder a la universidad, concretamente, un $60 \%$ menos. Es el mismo resultado que encontraron Peraita y Sánchez (1998), Rahona (2006) y Albert (2000), y lo explican en base a un efecto renta. Es decir, el hecho de presentar una mayor proporción de desempleados o de que haya menores de 16 años en el hogar significa disponer de menos recursos para afrontar una inversión en educación superior. Por último, el tamaño de la familia en cuanto a las personas que forman parte de ella también resulta significativo, de modo que cada miembro más en el hogar implica un aumento del $20 \%$ de probabilidades de demandar, contrariamente a lo que cabría esperar con un efecto renta. En conjunto, los factores que se refieren a los recursos económicos disponibles de los individuos parece que ejercen una influencia positiva en el hecho de solicitar estudios universitarios.

Los factores que se relacionan con aspectos más de tipo cultural, como observamos en la tabla 5, mantienen la influencia en la demanda que ya apuntaban otros análisis ya presentados. Los jóvenes con padres u otros miembros del hogar con estudios universitarios presentan mayores probabilidades de demandar estudios universitarios — tres veces más en el caso de los padres y casi el doble en el caso de que sean otros individuos del hogar los que posean estudios universitarios-. Aunque no podemos afirmar de forma definitiva si el mecanismo que influye en esta relación es de tipo cultural (habilidades, motivaciones) o económico (efecto renta), sí podemos asegurar que la influencia encontrada en estudios anteriores se mantiene para el año 2009. Así pues, con mayor probabilidad, los jóvenes que demandan estudios universitarios son, principalmente, de origen social alto, tanto por lo que se refiere a la educación, como desde el punto de vista profesional, y tienden a convivir en un entorno proclive a la universidad, además, en su hogar, cuentan con la presencia de otros miembros con estudios superiores. Ahora podemos afirmar que, con padres u otras personas que poseen estudios universitarios, las probabilidades de acceder a los niveles académicos superiores crecen fuertemente.

En cuanto a la variable sexo, se confirma el hecho de que las mujeres tienden a demandar estudios universitarios en mayor proporción, en concreto, presentan casi el doble de probabilidades que los hombres, lo que se puede explicar en base a la peor situación femenina en el mercado laboral, puesto que ellas necesitan una titulación universitaria para poder competir con los hombres en el mundo del trabajo (Mora, 1997; Merle, 2009), entre otros posibles factores. En conclusión, las variables referentes al origen social, incluso controladas por factores económicos, son las más importantes a la hora de explicar la demanda de educación universitaria; esto indica que, aunque las barreras económicas están muy presentes, las disposiciones y las motivaciones de clase social constituyen aún la principal barrera para acceder a los estudios superiores. 
Tabla 5. Estimación de coeficientes del modelo teórico inicial (modelo 1) y de la agregación del indicador de situación laboral (modelo 2). Variable dependiente: demanda de estudios universitarios

\begin{tabular}{|c|c|c|c|c|c|c|}
\hline \multirow[b]{2}{*}{ Variables independientes } & \multicolumn{3}{|c|}{ Modelo 1} & \multicolumn{3}{|c|}{ Modelo 2} \\
\hline & B & Sig. & $\operatorname{Exp}(B)$ & B & Sig. & $\operatorname{Exp}(B)$ \\
\hline Constante & $-0,397$ & * & 0,672 & 1,231 & ** & 3,424 \\
\hline Proporción de desempleados en el hogar & $-0,877$ & ** & 0,416 & $-2,168$ & ** & 0,114 \\
\hline Presencia de menores de 16 años en el hogar & $-0,905$ & ** & 0,405 & $-0,899$ & ** & 0,407 \\
\hline Tamaño del hogar (número de miembros) & 0,194 & ** & 1,214 & 0,104 & ** & 1,110 \\
\hline CSE hogar administrativos & $-0,832$ & ** & 0,435 & $-1,005$ & $\star \star$ & 0,366 \\
\hline CSE hogar cuenta propia & $-0,481$ & ** & 0,618 & $-1,072$ & ** & 0,342 \\
\hline CSE hogar obreros y agricultores & $-1,371$ & ** & 0,254 & $-1,849$ & * & 0,157 \\
\hline Educación padres. Nivel secundario & 0,417 & ** & 1,518 & 0,259 & * & 1,296 \\
\hline Educación padres. Nivel universitario & 1,131 & ** & 3,099 & 0,832 & ** & 2,298 \\
\hline $\begin{array}{l}\text { Presencia de otros miembros con estudios } \\
\text { superiores en hogar }\end{array}$ & 0,469 & ** & 1,599 & 0,400 & $\star *$ & 1,492 \\
\hline Mujer & 0,619 & ** & 1,857 & 0,573 & $\star *$ & 1,773 \\
\hline Trabaja a tiempo parcial & & & & $-0,506$ & ** & 0,603 \\
\hline Trabaja a tiempo completo & & & & $-1,715$ & ** & 0,180 \\
\hline Número de observaciones & & 2.492 & & & 2.492 & \\
\hline Significatividad del modelo (chi cuadrado) & & ** & & & ** & \\
\hline Porcentaje de predicciones correctas no acceso (0) & & $67,8 \%$ & & & $72,9 \%$ & \\
\hline Porcentaje de predicciones correctas acceso (1) & & $71,5 \%$ & & & $75,1 \%$ & \\
\hline Porcentaje de predicciones correctas & & $70,0 \%$ & & & $74,2 \%$ & \\
\hline R2 de Nagelkerke & & 0,275 & & & 0,368 & \\
\hline
\end{tabular}

* Coeficiente significativo al 0,05.

** Coeficiente significativo al 0,01 .

Punto de corte $=0,57$.

Fuente: elaboración propia a partir de datos de la EPA.

\subsection{La dimensión transicional}

En el modelo 2 de la tabla 5, se ha agregado el indicador de la relación con el mercado de trabajo. La primera observación es que este modelo es más explicativo que el primero: la R2 de Nagelkerke pasa de un 0,275 a un 0,368. La relación con el mundo laboral influye en la demanda de estudios universitarios (hipótesis 2), en el sentido de que los jóvenes que trabajan a tiempo completo presentan un $80 \%$ menos de probabilidades de demandar dicho nivel educativo respecto a los que no trabajan. Esta relación parece indicar que, a medida que se va estando inserto laboralmente, el interés o la capacidad para acceder a la educación superior es menor. Además, el efecto es mayor entre los que trabajan a tiempo completo que entre los que trabajan a tiempo parcial, con una disminución de las probabilidades del $80 \%$ i del $40 \%$, respectivamente.

También podría indicar que aquellos individuos que no quieran o no puedan demandar estudios universitarios se hayan «buscado ya la vida» en el 
Tabla 6. Estimación de coeficientes. Variable dependiente: demanda de estudios universitarios. Variable de control: situación residencial

\begin{tabular}{|c|c|c|c|c|c|c|}
\hline \multirow[b]{2}{*}{ Variables independientes } & \multicolumn{3}{|c|}{ Independizados } & \multicolumn{3}{|c|}{ No independizados } \\
\hline & B & Sig. & $\operatorname{Exp}(B)$ & B & Sig. & $\operatorname{Exp}(B)$ \\
\hline Constante & 1,984 & ** & 7,271 & 1,498 & ** & 4,472 \\
\hline Proporción de desempleados en el hogar & $-2,194$ & ** & 0,112 & $-1,912$ & ** & 0,148 \\
\hline Presencia de menores de 16 años en el hogar & $-2,013$ & ** & 0,134 & & n.s. & \\
\hline Tamaño del hogar (miembros) & $-0,352$ & * & 0,704 & $-0,150$ & * & 0,860 \\
\hline CSE hogar administrativos & $-1,128$ & ** & 0,324 & $-0,725$ & ** & 0,484 \\
\hline CSE hogar cuenta propia & $-1,812$ & * & 0,163 & $-0,618$ & * & 0,539 \\
\hline CSE hogar obreros y agricultores & $-2,491$ & ** & 0,083 & $-1,174$ & ** & 0,309 \\
\hline Educación padres. Nivel secundario & & n. s. & & 0,399 & * & 1,490 \\
\hline Educación padres. Nivel universitario & & n. s. & & 1,468 & ** & 4,339 \\
\hline Presencia de otros estudios superiores en hogar & 1,339 & ** & 3,816 & 0,581 & ** & 1,799 \\
\hline Mujer & 1,262 & ** & 3,533 & 0,618 & ** & 1,852 \\
\hline Trabaja a tiempo parcial & $-1,174$ & * & 0,309 & & n.s & \\
\hline Trabaja a tiempo completo & $-2,054$ & ** & 0,128 & $-1,581$ & ** & 0,206 \\
\hline Número de observaciones & & 27 & & & 1.868 & \\
\hline Significatividad del modelo (chi cuadrado) & & & ** & & ** & \\
\hline Porcentaje de predicciones correctas no acceso (0) & & $76,0 \%$ & & & $75,0 \%$ & \\
\hline Porcentaje de predicciones correctas acceso (1) & & $81,1 \%$ & & & $67,2 \%$ & \\
\hline Porcentaje de predicciones correctas & & $77,6 \%$ & & & $69,8 \%$ & \\
\hline R2 de Nagelkerke & & 0,51 & & & 0,304 & \\
\hline
\end{tabular}

* Coeficiente significativo al 0,05.

** Coeficiente significativo al 0,01.

n. s. Coeficiente no significativo.

Punto de corte: independizados $=0,31$; no independizados $=0,68$.

Fuente: elaboración propia a partir de datos de la EPA.

mundo laboral, por este motivo, encontramos tales diferenciales de probabilidad. En cualquiera de las dos interpretaciones, observamos que la relación con el mercado de trabajo es fundamental para dar cuenta de esta demanda educativa ${ }^{4}$ : la inserción laboral lleva a una menor probabilidad de demanda de estudios, y si además es a tiempo completo, esta relación es aún más relevante. En cuanto a los demás condicionantes, no se observan grandes variaciones respecto al modelo 1 .

Para la tercera hipótesis, se ha utilizado la variable de control situación residencial observando el modelo 2 , el cual se ha demostrado como más explicativo, para emancipados y no emancipados de forma diferenciada. Los resultados se recogen en la tabla 6 .

4. Hay que tener en cuenta que la variable relación con el mercado de trabajo puede indicar la situación una vez que el joven esté cursando estudios universitarios o los haya finalizado debido a la muestra considerada. Aún teniendo esto en cuenta, la relación es significativa, por lo que se constata que la relación con el mercado de trabajo tiene una relación real con la demanda de estudios universitarios. 
Si bien los jóvenes emancipados han demandado menos universidad que los que aún viven con los padres ( $32 \%$ y $68 \%$ de los que potencialmente pueden acceder, respectivamente), curiosamente, en los primeros, el nivel formativo de los padres no influye en la elección educativa. Esto quiere decir que los jóvenes que no viven con los padres demandan por igual, independientemente del origen formativo. De este modo, entre los jóvenes emancipados, el origen formativo deja de tener influencia, aunque aumenta el papel de la categoría socioeconómica del hogar como condicionante de la demanda.

Como vemos en la tabla 6 , las variables del contexto del hogar influyen más en los jóvenes emancipados, especialmente la existencia de menores (que probablemente serán sus hijos), lo cual nos muestra la importancia de las trayectorias vitales y familiares a la hora de acceder a la universidad. Es decir, el impacto sobre la variación de las probabilidades de los factores contextuales del hogar es mayor entre los emancipados y los que viven con los padres. El soporte que da la familia implica que los factores del hogar no influyan tanto como cuando se está emancipado.

En cuanto al sexo del individuo, las mujeres presentan mayores probabilidades de acceder a la universidad si están emancipadas (tres veces más que los hombres) que si no lo están (casi el doble que los hombres). Finalmente, la variable que indica la situación respecto al mercado de trabajo también influye más en los jóvenes emancipados, en el sentido de que, si están trabajando, presentan una menor demanda de acceso a la universidad. Reduce las probabilidades en un $90 \%$, respecto al $80 \%$ entre los que viven aún con los padres.

Resumiendo, la emancipación residencial implica una menor demanda de estudios universitarios, probablemente debido a que, dentro de esta categoría, se encuentran trayectorias de maternidad o de inserción laboral temprana, las cuales diluyen las motivaciones y las posibilidades de acceder a los niveles académicos superiores (un 51\% de los emancipados trabaja a tiempo completo, respecto a un $44 \%$ de los no emancipados). Así mismo, cabría pensar que los recursos disponibles para costearse los estudios son menores entre los emancipados y, de hecho, en el análisis, observamos incluso que los recursos económicos son más decisivos entre los emancipados. Por otro lado, los factores referentes al origen familiar pierden fuerza.

\section{Conclusiones}

A partir de los estudios que se han llevado a cabo en el Estado español, construimos un modelo teórico inicial con los principales condicionantes analizados, para observar si se reproducían los mismos resultados para el año 2009 y podríamos formar un modelo de demanda educativa suficientemente explicativo para este contexto. Los resultados muestran que las siete hipótesis se confirman, aunque la que se refiere al tamaño del hogar actúa en dirección contraria a lo esperado. Esto puede atribuirse al hecho de que, en el modelo 1 y 2 , se consideran conjuntamente y de forma excepcional por la base utilizada a emancipados y a no emancipados, teniendo en cuenta que los emancipados 
(hogares más pequeños) demandan menos estudios universitarios. Hay una relación espuria, que es necesario controlar. Si observamos los resultados de este factor en el análisis que separa a ambos colectivos (tabla 6), la relación sí que es la esperada en ambos, por lo que confirmamos la hipótesis inicial. Al tratarse de una base de datos que nos permite incluir en el análisis a los jóvenes emancipados, puede entenderse que, en el modelo 1, esta variable dé un resultado contrario a los análisis realizados con otras bases de datos.

El hecho de observar cómo los factores que indican los recursos económicos disponibles en el hogar son significativos, implica que estos constituyen aún una barrera importante en la demanda de estudios universitarios, lo que nos plantea interrogantes sobre el futuro de la universidad con el aumento de los precios públicos de matriculación en el curso 2012-2013. ¿Pueden estos condicionantes económicos aumentar su influencia en un futuro próximo?

El modelo teórico inicial propuesto es suficientemente explicativo (R2 de Nagelkerke de $0,27^{5}$ ) como para reconocer su significatividad sociológica. Los recursos disponibles del individuo, el origen formativo familiar y las motivaciones derivadas del sexo y de la convivencia con otras personas con estudios universitarios son, para 2009, aspectos que influyen a la hora de elegir demandar o no estudios universitarios. Hay que destacar que el origen social sigue siendo muy relevante en el modelo, aunque se haya controlado mediante indicadores referentes a los recursos económicos. Esto indica la importancia de las motivaciones de clase a la hora de construir la trayectoria educativa más allá de sus recursos y plantea la necesidad de tratar que la universidad sea concebida para todos los grupos sociales como una opción factible.

Una vez analizado el modelo teórico inicial, introducimos en el análisis otras variables que hacen referencia a aspectos transicionales, a aspectos de la trayectoria del individuo que condicionan las elecciones presentes y futuras. Entre la juventud analizada en un modelo de demanda determinado, no toda ha dibujado la misma trayectoria vital o un mismo itinerario como joven. No constituye un grupo homogéneo y, por lo tanto, es relevante utilizar variables que indiquen qué itinerario vital ha seguido para comprobar si funcionan como discriminantes de la demanda educativa, más allá de los factores tradicionalmente observados.

Así, consideramos la relación con el mercado de trabajo como un factor más a tener en cuenta en el modelo de elección. Observamos que, efectivamente, los jóvenes que están trabajando a tiempo completo demandan estudios universitarios en un $80 \%$ menos que los que no están trabajando. Esto significa, por un lado, que una proporción relevante de universitarios no trabaja y, por otro, que aquellos jóvenes que tienen un trabajo poseen pocos incentivos para seguir estudiando. ¿Hasta qué punto esto se debe a una difícil conciliación entre el mundo académico y el laboral? Un sistema universitario equitativo y competitivo debería poder ser un reclamo también para los jóvenes que ya están

5. Un nivel parecido al encontrado en otros estudios como el de De Pablos y Gil (2007) o Rahona (2006), aunque, obviamente, con modelos de demanda y poblaciones diferentes. 
trabajando, que acostumbran a ser de orígenes familiares medios o bajos, o, al menos, permitir la compaginación entre estudios y trabajo, para no disponer sólo de estudiantes a tiempo completo (casi el 60\% de los demandantes de educación universitaria no están empleados).

Una segunda dimensión transicional que analizamos hace referencia a la transición residencial. Nos preguntamos si dicha situación introduce variaciones en el modelo de demanda educativa planteado. La configuración de los factores en el modelo de demanda entre emancipados y no emancipados se modifica: los condicionantes económicos ganan peso entre los primeros, cosa esperable, ya que no gozarían del soporte familiar directo y, curiosamente, los condicionantes de origen familiar pierden fuerza. El hecho de emanciparse reduce las probabilidades de haber demandado estudios universitarios y modifica el peso de los factores del modelo de demanda.

Resumiendo, los hechos de sufrir dificultades económicas, de haber accedido al mercado laboral o de haberse independizado pueden constituir un obstáculo para la demanda de estudios universitarios, más allá del efecto neto del origen social. Cabe destacar la importancia de la dimensión transicional a la hora de enriquecer este análisis, ya que no todos los jóvenes se encuentran en el mismo itinerario vital, lo que introduce complejidad en el modelo de demanda educativa y plantea retos políticos importantes para que el paso por la universidad no sea sólo el camino de unos perfiles juveniles concretos. Una política de becas adecuada puede diluir los condicionantes económicos y una correcta compaginación del mundo laboral y el mundo educativo, lo que permitirá que ciertos itinerarios juveniles no constituyan una barrera por sí mismos a la hora de demandar estudios universitarios. En cualquier caso, será necesario seguir el análisis de estos efectos, especialmente con metodologías tipo panel más adecuadas para nuestros objetivos.

\section{Referencias bibliográficas}

ARIÑo, Antonio y Llopis, Ramón (2011) (eds.). ¿Universidad sin clases?: Condiciones de vida de los estudiantes universitarios en España (Eurostudent IV). Madrid: Ministerio de Educación.

Albert, Cecilia (2000). «Higher Education Demand in Spain: The Influence of Labour Market Signals and Family Background». Higher Education [en línea], 40 (2), 147-162. <http://dx.doi.org/10.1023/A:1004070925581>.

Baudelot, Christian y Establet, Roger (1987). La escuela capitalista. México: Siglo XXI.

BeCKer, Gary (1975). Human capital [en línea]. Nueva York: NBER. $<$ http://dx.doi.org/10.7208/chicago/9780226041223.001.0001>.

Behrman, Jere y Taubman, Paul (1989). «Is schooling mostly in the genes?: Nature - nurture descomposition using data on relatives». Journal of Political Economy [en línea], 97 (6), 1425-1446. <http://dx.doi.org/10.1086/261661>.

Bernstein, Basil (1988). Clases, códigos y control. Madrid: Arkal. 
Bourdieu, Pierre y Passeron, Jean-Claude (1990). Reproduction in Education, Society, and Culture. Londres: Sage in association with Theory Culture \& Society Dept. of Administrative and Social Studies Teesside Polytechnic, 1977.

Bowles, Sam y Gintis, Herb (2002). «The inheritance of inequality». Economic Perspectives [en línea], 16 (3), 3-30. <http://dx.doi.org/10.1257/089533002760278686>.

Calero, Jorge (1996). Financiación de la Educación Superior en España: Sus implicaciones en el terreno de la equidad. Bilbao: Fundación BBV.

Carabaña, Julio (2004). «Una estimación del grado en que el número de hermanos deprime el nivel de estudios». En: Rospir, Juan Ignacio; Torre, Isabel de la y Durán, María Ángeles (coords.). Reflexiones sociológicas: Libro homenaje a José Castillo Castillo. Madrid: CIS.

- (2005). «Educación y movilidad social». En: Navarro, Vicenç (coord.). El Estado de Bienestar en España. Madrid: Tecnos.

Casal, Joaquim; García, Maribel; Merino, Rafael y Quesada, Miguel (2006a). «Aportaciones teóricas y metodológicas a la sociología de la juventud desde la perspectiva de la transición». Papers: Revista de Sociologia, 79, 21-48.

- (2006b). "Changes in forms of transition in contexts of informational capitalism». Papers: Revista de Sociologia, 79, 195-223.

EsCribÀ, Abel (2006). «Estructura familiar, estatus ocupacional y movilidad social intrageneracional en España». Revista Internacional de Sociología [en línea], 64 (45), 145-170. <http://dx.doi.org/10.3989/ris.2006.145.19>.

FACHelli, Sandra (2009). Nuevo modelo de estratificación social y nuevo instrumento para su medición: El caso argentino [en línea]. Barcelona. <http://hdl.handle.net/10803/5149>.

Fachelli, Sandra; Torrents, Dani y Navarro-Cendejas, José (2014). "¿La universidad española suaviza las diferencias de clase en la inserción laboral?». Revista de Educación [en línea], 364, 119-144. <http://dx.doi.org/10.4438/1988-592X-RE-2014-364-257>.

Furlong, Andy; Cartmel, Fred y Bigart, Andy (2006). "Choice biographies and transitional linearity: Re-conceptualising modern youth transitions». Papers: Revista de Sociologia, 79, 225-239.

Gil, María; Pablos, Laura de y Martínez, María (2010). «Los determinantes socioeconómicos de la demanda de educación superior en España y la movilidad educativa intergeneracional». Hacienda Pública Española, 193, 75-108.

González, Beatriz y DÁvila, Delia (1998). «Economic and cultural impediments to University education in Spain». Economics of Education Review [en línea], 17 (1), 93-103. <http://dx.doi.org/10.1016/S0272-7757(97)00010-1>.

Instituto NaCional de Estadística (2010). Módulo sobre la incorporación de los jóvenes en el mercado laboral, asociado a la Encuesta Comunitaria de Fuerza de Trabajo del año 2009. Informe final. Madrid: INE.

Jackson, Michelle (2013) (ed.). Determined to Succeed?: Perfomance versus Choice in educational Attainment. California: Standford University Press.

KodDe, David (1986). "Uncertainty and the Demand for Education». Review of Economics and Statistics [en línea], 68 (3), 460-67. <http://dx.doi.org/10.2307/1926023>.

López, Mercedes (1995). La elección de una carrera típicamente femenina o masculina. Madrid: Ministerio de Educación. 
López-Roldán, Pedro y Lozares, Carlos (2000). Anàlisi multivariable de dades estadistiques. Barcelona: Universitat Autònoma de Barcelona.

Merino, Rafael y García, Maribel (2007). Itineraris de formació i inserció laboral dels joves a Catalunya. Barcelona: Fundació Jaume Bofill.

Merle, Pierre (2009). La démocratisation de l'enseignement. París: La Découverte.

Mora, José-Ginés (1997). «Equity in Spanish Higher Education». Higher Education [en línea], 33, 233-249. <http://dx.doi.org/10.1023/A:1002950227350>.

Moulton, Brent (1990). "An illustration of a pitfall in estimating the effects of aggregate variables on micro units». The Review of Economics and Statics [en línea], 72 (2), 334-338. <http://dx.doi.org/10.2307/2109724>.

Pablos, Laura de y Gil, María (2007). «Análisis de los condicionantes socioeconómicos del acceso a la educación superior». Presupuestos y Gasto Público, 48, 37-57.

Peraita, Carlos y Sánchez, Manuel (1998). "The effect of family background on children's level of schooling attainment in Spain». Apllied Economics [en línea], 30 (10), 1327-1334. <http://dx.doi.org/10.1080/000368498324940>.

Planas, Jordi y FaCHelli, Sandra (2010). Les universitats catalanes, factor d'equitat $i$ de mobilitat profesional. Barcelona: AQU.

Pollock, Gary (2009). "Trajectòries juvenils i mobilitat social». Revista Àmbits, 41, 36-42.

Rahona, Marta (2006). «La influencia del entorno socioeconómico en la realización de estudios universitarios: Una aproximación en el caso español en la década de los noventa». Hacienda Pública Española / Revista de Economía Pública, 178 (3), 55-80.

- (2009). «Equality of opportunities in Spanish higher education». Higher Education [en línea], 58, 285-306. <http://dx.doi.org/10.1007/s10734-008-9194-5>

Soler, Roger; Serracant, Pau; Salvadó, Antoni y Miret, Pau (2008). Enquesta a la joventut de Catalunya 2007. Barcelona: Secretaria de Joventut.

STAFF, Jeremy (2007). «Educational and work strategies from adolescence to early adulthood: Consequences of educational attainment». Social Forces [en línea], 85 (3), 1169-1194. <http://dx.doi.org/10.1353/sof.2007.0057>.

Velázquez, Francisco (2004) (dir.). Anuario Estadistico de la Universidad Española 2004. Madrid: Ministerio de Educación y Ciencia. 